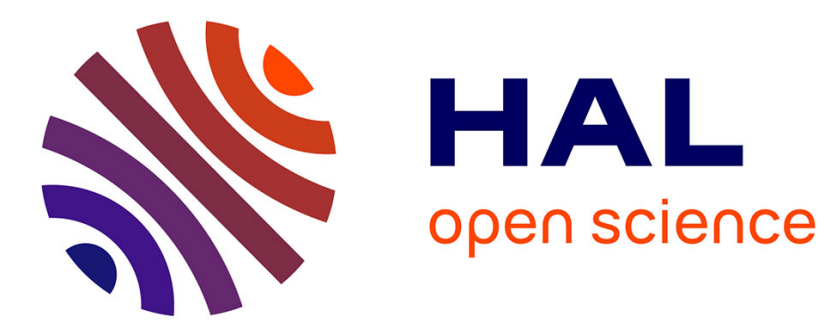

\title{
The Influence of Relative Disparity and Planar Motion Velocity on Visual Discomfort of Stereoscopic Videos
}

Jing Li, Marcus Barkowsky, Patrick Le Callet

\section{To cite this version:}

Jing Li, Marcus Barkowsky, Patrick Le Callet. The Influence of Relative Disparity and Planar Motion Velocity on Visual Discomfort of Stereoscopic Videos. International Workshop on Quality of Multimedia Experience QoMEX, Sep 2011, Mechelen, Belgium. pp.1-6. hal-00637497

\section{HAL Id: hal-00637497 https://hal.science/hal-00637497}

Submitted on 2 Nov 2011

HAL is a multi-disciplinary open access archive for the deposit and dissemination of scientific research documents, whether they are published or not. The documents may come from teaching and research institutions in France or abroad, or from public or private research centers.
L'archive ouverte pluridisciplinaire HAL, est destinée au dépôt et à la diffusion de documents scientifiques de niveau recherche, publiés ou non, émanant des établissements d'enseignement et de recherche français ou étrangers, des laboratoires publics ou privés. 


\title{
THE INFLUENCE OF RELATIVE DISPARITY AND PLANAR MOTION VELOCITY ON VISUAL DISCOMFORT OF STEREOSCOPIC VIDEOS
}

\author{
Jing Li, Marcus Barkowsky and Patrick Le Callet \\ LUNAM Université, Université de Nantes, IRCCyN UMR CNRS 6597 \\ (Institut de Recherche en Communications et Cybernétique de Nantes) \\ Polytech Nantes, rue Christian Pauc BP 5060944306 Nantes Cedex 3, France \\ \{jing.li2,marcus.barkowsky,patrick.lecallet\}@univ-nantes.fr
}

\begin{abstract}
The vergence-accommodation conflict, excessive screen disparity, binocular distortions and the motion component in stereoscopic videos are considered as main factors that may induce visual discomfort. In our previous study which was based on the experts-only experiment, we also found that the large relative disparity between the foreground and background and the fast planar motion were more likely to induce visual discomfort. In this study, we conducted the same subjective experiment but on non-expert observers. The subjective experiment results coincided with our previous findings. The two objective visual discomfort models developed in our previous study have been evaluated and showed high correlation with subjective data. Finally, we found that the observers could be classified into different clusters according to their visual discomfort sensitivity to the velocity or the relative disparity. For some observers, the velocity is the predominant factor that may induce visual discomfort; some consider that the relative disparity is the key factor, and some are sensitive to both the velocity and relative disparity.
\end{abstract}

Index Terms - Stereoscopic videos, velocity, relative disparity, visual discomfort, observer classification

\section{INTRODUCTION}

Visual discomfort and visual fatigue are frequent complaints of the viewers after watching stereoscopic videos. It may be one of the critical factors that impede the wide application of 3-D technology on some industries, especially cinema, television broadcast system and video games. Thus, it is necessary to investigate what factors may cause visual discomfort and then construct a visual discomfort model which can be used as the guidance for the creation of 3-D production to avoid inducing visual discomfort.

One of the widely accepted main reasons that may cause visual discomfort is the vergence-accommodation conflict [1]. The discrepancy that the eyes converge to the virtual object which is behind or in front of the screen while the accommodation is performed at the screen plane may induce visual discomfort. Kooi and Toet [2] investigated the influence of imperfections in binocular image pairs on visual discomfort. They indicated that for statistic stereoscopic images, the vertical disparity, crosstalk, and blur were mainly factors that could induce visual discomfort. Besides, research on visual discomfort induced by motion has been conducted as well. In 2002, Yano et al. found that a local minimum of visual comfort appeared for both high degree of parallax and amount of motion [3]. In 2004, Yano et al. [4] pointed out that the visual fatigue occurred when the stereoscopic images involved an in-depth motion component even if they were displayed within the range of depth of field. In 2006, Speranza et al. [5] concluded that motion in depth, i.e., the magnitude of binocular disparity varying over time, could play an important role in visual discomfort, and it might be more important in determining visual discomfort than the absolute magnitude of the binocular disparity. Recently, Lee et al. [6] investigated the visual discomfort induced by fast motion of salient objects in a stereoscopic video. They estimated the visual discomfort thresholds for in-depth, horizontal and vertical motion velocities at different depth and presented a visual comfort model which was based on the salient motion features.

In our previous study [7], we investigated the relationship between visual discomfort and the planar motion at different depth levels. It was shown that the relative disparity between the background and foreground played an important role in determining visual discomfort, and the planar motion velocity also had influence on the visual discomfort. However, this study was based on the experts-only subjective experiment results with only 10 observers, which may not give a comprehensive conclusion for the non-expert observers.

In this study, we conducted a similar subjective experiment to our previous study but with non-expert observers to verify our previous findings. Then, we tested the consistency of the objective visual discomfort model which was constructed in previous study on both the experts and non-experts subjective experiment data. Meanwhile, through the analysis of each observer's agreement on the global subjective experiment results, we found that there were different groups of observer responses which gave different opinions on the effects of relative disparity and velocity on visual discomfort.

This paper is organized as follows. In section 2, the experimental setup is presented. In section 3, the experimental results are analyzed, including the comparison with our previous conclusions, the evaluation of the Bradley-Terry models on the data analysis, the evaluation of the performance of the objective visual discomfort model and finally, the classification of the observers. Section 4 concludes the paper.

\section{EXPERIMENTAL SETUP}

As this study is meant to complement the subjective experiment which we have conducted before, the experimental setup was the same as previous except for the non-expert viewers, the number of trials for each viewer and the time limitation for displaying the stimuli. More details about these differences can be found in section 2.4 to 2.6 . 


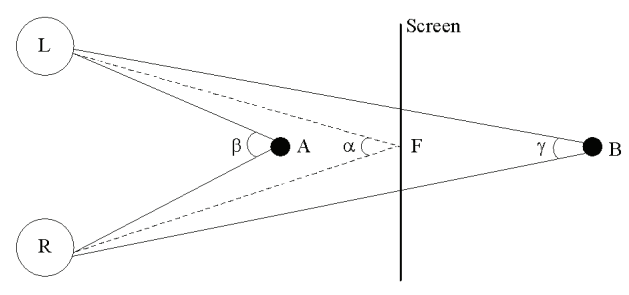

Fig. 1. The definition of the binocular angular disparity, where $\mathrm{F}$ is the fixation point.

\subsection{Experimental design}

The main task in this study is to investigate the effects of disparity and planar motion velocity on visual discomfort. Thus, five binocular disparity levels and three velocity levels are selected. Three of the binocular disparity levels are within the comfortable viewing zone [8], which is based on the depth of focus (DOF). A value of \pm 0.2 Diopter is suggested. Two are outside it. These disparities can be expressed in degrees of visual angle [9], as shown in Fig. 1. The binocular angular disparity can be calculated by the following equations 1 and $2, \phi_{A}$ and $\phi_{B}$ are binocular angular disparities for $\mathrm{A}$ and $\mathrm{B}$. Note that a positive value represents the crossed disparity, such as the point $\mathrm{A}$; a negative value represents the uncrossed disparity, such as the point B. In this study, the five angular disparity levels were $0, \pm 0.65$, and \pm 1.3 degree, assuming that the interpupillary distance was $65 \mathrm{~mm}$ and the viewing distance was $90 \mathrm{~cm}$.

$$
\begin{aligned}
& \phi_{A}=\beta-\alpha \\
& \phi_{B}=\gamma-\alpha
\end{aligned}
$$

Three velocity levels which represent slow, medium and fast are chosen. All of the velocities are within the range in which the viewers can pursue the objects smoothly without making catch-up saccades, which is suggested to reside below 30 degree/s [10]. More details are shown in section 2.2 .

\subsection{Stimuli}

Computer-generated stereoscopic sequences were used in this study to avoid the influence of other factors on visual discomfort. The stereoscopic sequences consisted of a left-view and a right-view image which were displayed by the MATLAB psychtoolbox [11]. Each image contained a foreground and a background. A black Maltese cross with $480 \times 480$ pixels was used as the foreground object as it contained both high and low spatial frequency components. This was supposed to limit the influence of one particular spatial frequency in the experiment. The object moved along a circular trajectory with center point at the center of the screen, and a radius of 300 pixels with the viewing angle of 10.13 degree. The reason to choose a circle as the trajectory was that it could avoid the step impulse that came from a sudden change of the motion direction, which may cause unexplained effects of visual discomfort. As the trajectory was a circle, the velocity was expressed in degree/s. The three velocity levels were $71.8,179.5$ and 287.2 degree/s, which represented slow, medium and fast, respectively. The motion direction was anti-clockwise. The background was placed at a fixed position which was with the angular disparity

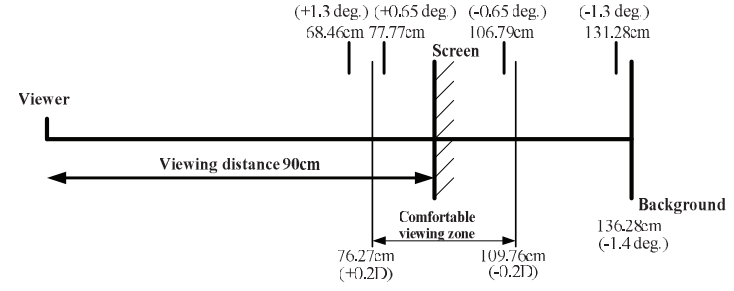

Fig. 2. The relationship of the foreground and the background position and the comfortable viewing zone.

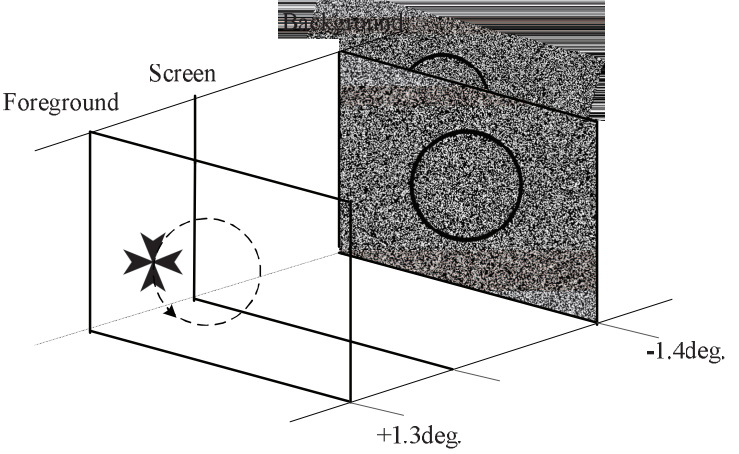

Fig. 3. An example of a stereoscopic image in the experiment. The foreground object is moving at the depth plane with a disparity of 1.3 degree. The background is placed at the depth plane with a disparity of -1.4 degree. The motion direction of the Maltese cross is anti-clockwise.

of -1.4 degree. It was generated by adding salt \& pepper noise on a black image, and then filtered by a circular averaging filter. This kind of background could preclude all of the monocular cues on stereopsis. Additionally, a black circle which was the same as the moving track of the object was placed on the background to give the viewers a reference of the trajectory. So, for viewers, the stimuli appeared to be composed of two parts: the salt\&pepper-like background with a black circle on it, and a moving Maltese cross on a depth plane with a certain velocity.

Fig.2 shows the disparities used in the stimuli and their relationship with comfortable viewing zone. As there were 3 levels of velocity and 5 levels of angular disparity, there were totally 15 stimuli for the experiment. An example of the stimuli is shown in Fig.3, in which the foreground object is placed in front of the screen with an angular disparity of 1.3 degree.

\subsection{Apparatus}

The stereoscopic sequences were displayed on a Dell Alienware AW2310 23-inch 3-D LCD screen $(1920 \times 1080$ full HD resolution, $120 \mathrm{~Hz}$ ), which featured $0.265-\mathrm{mm}$ dot pitch. The display was adjusted for a peak luminance of $50 \mathrm{~cd} / \mathrm{m}^{2}$ when viewed with the active shutter glasses. The graphics card of the PC was an NVIDIA Quadro FX 3800. Stimuli were viewed binocularly through the NVIDIA active shutter glasses (NVIDIA 3D vision kit) at a distance of about $90 \mathrm{~cm}$, which was approximately three times of the 
picture height. The peripheral environment luminance was adjusted to about $44 \mathrm{~cd} / \mathrm{m}^{2}$. When seen through the eye-glasses, this value corresponded to about $7.5 \mathrm{~cm} / \mathrm{m}^{2}$ and thus to $15 \%$ of the screen's peak brightness as specified by ITU-R BT.500 [12].

\subsection{Viewers}

Forty-five viewers participated in this subjective experiment. Twenty-one are male, twenty-four are female. They are all non-expert in subjective experiment, image processing or 3D related field. Their ages ranged from 18 to 44 years old with average age 24. All have either normal or corrected-to-normal visual acuity. The visual acuity test was conducted with a Snellen Chart for both far and near vision. The Randot Stereo Test was applied for stereo vision acuity check, and Ishihara plates were used for color vision test. All of the viewers passed the pre-experiment vision check.

\subsection{Assessment Method}

In this study, the paired-comparison method was chosen as it is a well-known method in the field of psychophysics [13]. In the experiment, the viewers watched a pair of stimuli at one trial, and then they were asked to select the one which made them more uncomfortable. A total of $\left(\begin{array}{c}15 \\ 2\end{array}\right)=105$ pairs were presented in each individual subjective experiment. The presentation order of stimuli in one paired comparison was different for odd numbered and even numbered observers. For example, observers with even numbers will watch stimulus A first, then stimulus B. For odd numbered observers, this order is inversed. This is used to balance the presentation order. The presentation order for voting the whole 105 paired comparisons was randomly permuted for each viewer.

\subsection{Procedure}

The subjective experiment contained a training session and a test session. In the training session, there were five pairs of stimuli. At the beginning, the viewers were told that they would watch a series of stereoscopic motion images. They were asked not to stare at the moving object all the time, but watch the whole view of the stereoscopic sequence under test. Then, they should select the one which made them feel more uncomfortable, concerning e.g., eye strain, headache, etc. The viewers use two keys to switch between the pair of stimuli on one screen. There was a minimum time limit of 5 seconds for the display of stimuli, which means each observer had to watch each of the stimuli at least 5 seconds before making their decision by pressing a specified button. After the explanation of the experiment, the viewers were asked to do the test by themselves. During the training session, all questions of the viewers were answered. We ensured that after the training session, all of the viewers knew about the process and task of this experiment clearly.

In the main test session, the task and procedure were the same as the training session except 105 pairs of stimuli were compared. As the duration of the whole test was different due to the individual difference of each viewer, and to avoid visual fatigue caused by long time watching affecting the experimental results, the viewers were asked to have a 10 minutes break after half of the test samples.

\section{EXPERIMENTAL RESULTS}

The subjective paired-comparison results can be expressed by a three-dimensional matrix $M(i, m, n) . \quad M(i, m, n)=1$ if the $i_{t h}$ viewer selected stimulus $m$ when compared with stimulus n. $G(m, n)$ is the global subjective experiment results which represents the total number of times that stimulus $m$ is selected over stimulus n. It can be calculated by $G(m, n)=\sum_{i} M(i, m, n)$. Thurstone-Mosteller (TM) model (Thurstone's Law of Comparative Judgment, Case V) [14][15] and Bradley-Terry (BT) model [16] are two well-known paired comparison models which can convert the paired comparison data to psychophysical scale rating. In our previous study, the TM model was used to analyze the raw data owing to its origins in psychophysics. However, Bradley-Terry model is more developed mathematically. It can provide not only a tractable estimators for scales, but also confidence intervals and a series of hypothesis test. Thus, in this study we chose the BT model to analyze both the previous experts' data and the non-experts' data. More details about the comparison between these two models can be found in [17]. The program used in this study for BT model is available in [18].

\subsection{Analysis on experts and non-experts data}

As there were a foreground object and a background in the stimulus, the relative disparity between the foreground object and the background was used to analyze their effects on visual discomfort. The binocular angular disparity of the background was -1.4 degree, thus the 5 relative angular disparity levels of the foreground object were $0.1,0.75,1.4,2.05,2.7$ degree. The BT scores for visual discomfort from experts and non-experts data are shown in Fig.4.

Both the experts and non-experts BT scores for 15 stimuli give the same conclusion as what we have found in the previous experts-only study by utilizing TM model. It shows that the visual discomfort increases with the relative angular disparity rather than the absolute angular disparity of the object. The influence of the vergence-accommodation conflict seems to be quite small under this experimental setup. It might be explained by the existence of the background and the moving foreground. There would be two vergence points in the stimulus for the viewers. When watching the stimulus, the viewers' attention may switch between the two objects. The larger of the depth distances between the visual attention points, the larger the abrupt change of the amount of vergence-accommodation mismatch when switch from one object to another, which might be seen as a reason that induces the visual discomfort.

The experimental results also clearly indicated that the perceived visual discomfort increases with velocity. This conclusion is in accordance with our previous study. And it is also consistent with the results that Lee et al. gave in [6] recently although the planar motion directions are different. They pointed out that the visual discomfort increased with the velocity of horizontal and vertical motion while a circular motion was used in our test.

In a practical application of our study, it may be concluded that for stereoscopic motion images, the depth range between foreground and background for fast motion sequences should be significantly reduced and for slow motion sequences, the depth range could be increased. 

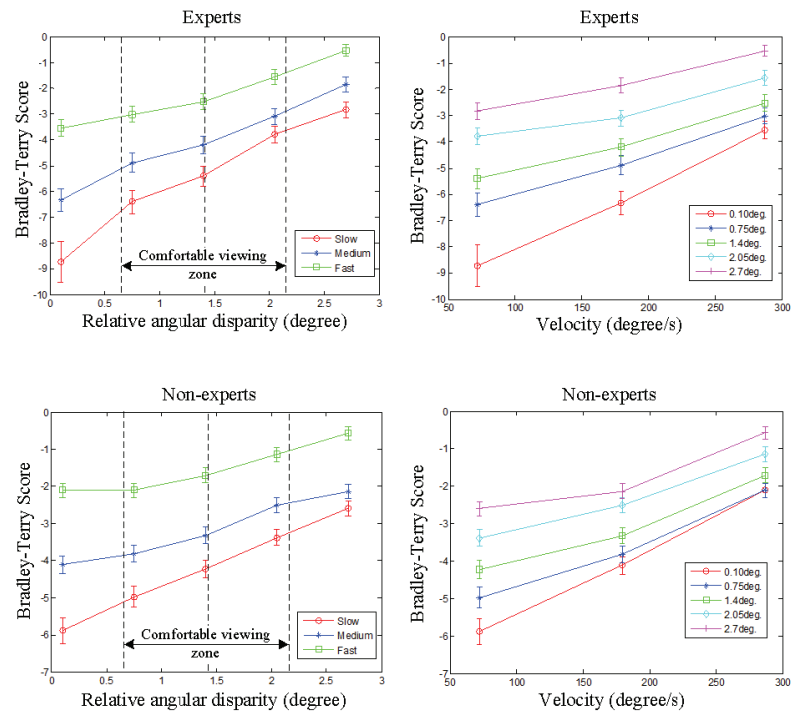

Fig. 4. BT scores for visual discomfort. The top two figures are experts results. The bottom two figures are non-experts results. The different lines in the left figures represent the different velocity levels, where slow, medium and fast represent 71.8, 179.5 and 287.2 degree/s. The outer two dashed lines represent the upper and lower limits of the comfortable viewing zone, which are 0.66 and 2.14 degree. The dashed line in the middle represents the position of screen plane. The different lines in the right figures represent the different relative angular disparity levels. The error bars are the $95 \%$ confidence interval.

\subsection{Evaluation on the BT scores}

In this part, we check the consistency of the BT scores of all stimuli with the global and individual subjective paired comparison results.

For better illustration, some definitions of the matrix which will be used in the consistency test are given. $B_{B-T}(m, n)$ is introduced to represent if the BT score of stimulus $\mathrm{m}$ is higher than that of stimulus $\mathrm{n}$. Thus, $B_{B-T}$ is a binary matrix without considering the diagonal elements. To compare the matrix $B_{B-T}$ with the global subjective experiment results, a binary matrix which represents the global subjective experiment results is needed and can be generated in the following way. Firstly, the probability matrix $P$ is calculated where $P(m, n)$ represents the probability that the stimulus $m$ is selected over stimulus $n$. Then, the values in $P$ which are below the threshold 0.5 are set to 0 and above the threshold to 1 . This binary matrix is expressed by $B_{O b s}$. Two evaluation methods were used to check the agreement of the BT scores with the subjective experiment results. The first one is the "Consistency test", which means using Student's-t-Test to check if $B_{B-T}(m, n)$ and $B_{O b s}(m, n)$ with $m<n$ were obtained from a Gaussian process with a common mean value. In our experiment, the Student's-t-Test was performed at $5 \%$ significance level. Secondly, an "Agreement test" was conducted both on the global and individual subjective experiment results, which means calculating the proportion that the value in each position of $B_{B-T}$ was the same with the corresponding value in $B_{O b s}$ and $M$ matrix of each observer.

The "Consistency test" and the "Agreement test" results for both experts and non-experts are shown in Table I, each observer's agreement on the BT scores are shown in Fig.5. Generally speaking, the results indicated that the BT scores fit well with the subjective
Table I. THE CONSISTENCY AND AGREEMENT TEST RESULTS

\begin{tabular}{|c|c|c|c|c|}
\hline \multirow{3}{*}{ Observers } & \multirow{3}{*}{$\begin{array}{c}\text { Consistency } \\
\text { Test }\end{array}$} & \multicolumn{3}{|c|}{ Agreement Test } \\
\hline & & \multirow{2}{*}{ Global } & \multicolumn{2}{|c|}{ Individual } \\
\hline & & & Mean & Std. \\
\hline Experts & pass & 0.9619 & 0.7917 & 0.0832 \\
\hline Non-experts & pass & 0.9714 & 0.8142 & 0.0909 \\
\hline
\end{tabular}
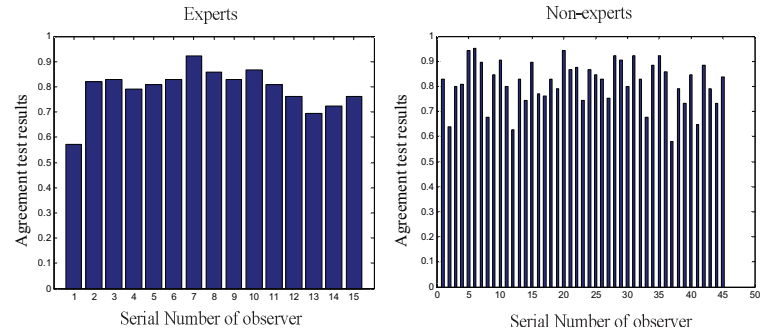

Fig. 5. The individual "Agreement test" results for both experts and non-experts data with the BT scores.

experiment results. However, it could be found that for some observers, their "Agreement test" results were lower, which means their opinions might differ from the global observers' opinion. Based on this analysis, it's necessary to cluster the observers as several classes in which they have the similar opinions. This will be investigated in section 3.4.

\subsection{Evaluation of the objective visual discomfort models}

In our previous study, we proposed two models for visual discomfort which was based on the TM score from experts-only subjective experiment data[7]. The Model 1 is expressed as:

$$
Q=a_{1} \cdot v+a_{2} \cdot d+a_{3}
$$

and Model 2 is expressed as:

$$
Q=b_{1} \cdot d+b_{2} \cdot v+b_{3} \cdot d \cdot v+b_{4}
$$

where $Q$ represents visual discomfort, $v$ is the velocity (degree/s) and $d$ is the relative angular disparity (degree), the predicted coefficients for the two models were $0.0018,0.2102,-0.0477$ for $a_{1}, a_{2}$ and $a_{3}$, and $0.3110,0.0026,-0.0006,-0.1888$ for $b_{1}, b_{2}, b_{3}$ and $b_{4}$, respectively.

In this study, both of the objective models will be evaluated by comparing the predicted scores with the BT scores. Three metrics are used as the evaluation criterions: (1) Pearson linear correlation coefficient (CC), which provides an evaluation of prediction accuracy. (2) Spearman rank-order correlation coefficient (ROCC), which is considered as a measure of the prediction monotonicity. (3) Root mean squared error (RMSE), which reflects the validation of prediction. The evaluation results are shown in Table II.

It can be concluded that the predicted visual discomfort scores from both of the models correlate quite well with the BT visual discomfort scores. Model 2 performs slightly better than Model 1. Both of the models can be used as index for the stereoscopic image related researches. 
Table II. The Performance of Model 1 And 2 ON The EXPERTS AND NON-EXPERTS BT SCORES

\begin{tabular}{ccccccc}
\hline \hline \multirow{2}{*}{ Model } & \multicolumn{3}{c}{ Experts } & \multicolumn{3}{c}{ Non-experts } \\
\cline { 2 - 7 } & CC & ROCC & RMSE & CC & ROCC & RMSE \\
\hline 1 & .9876 & .9750 & .0406 & .9489 & .9000 & .0788 \\
2 & .9949 & .9929 & .0257 & .9697 & .9286 & .0605 \\
\hline \hline
\end{tabular}
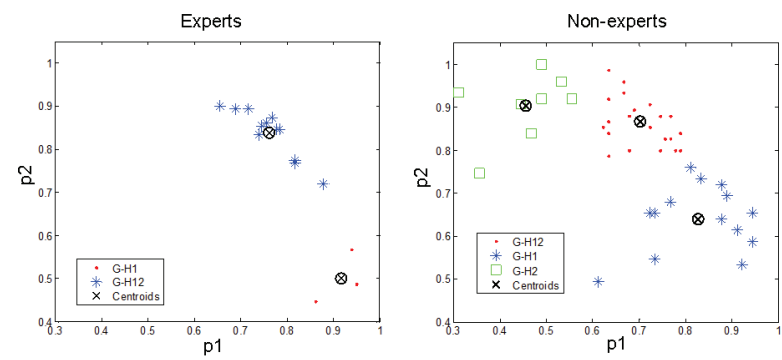

Fig. 6. The clustering results for experts and non-experts observers. X-axis represents the agreement on "relative disparity is the predominant factor" and y-axis represents the agreement on "velocity is the predominant factor".

\subsection{Classification of the observers}

In section 3.2 it has already been stated that there were some observers who had different opinions from the global subjective experiment results. Thus, it may be interesting to classify them into different groups and analyze the different influences of relative disparity and velocity on different observers.

The relative disparity and velocity are two factors that may induce visual discomfort in our study. Thus, the analysis of which factor is dominant in determining the visual discomfort is conducted on each observer. There are two hypotheses in this analysis. One is "the relative disparity is predominant" and the other is "the velocity is predominant". Then, the proportion of each observer voting for the stimulus whose relative disparity is larger is calculated for Hypothesis 1, expressed as $p 1$. And the proportion of voting for the stimulus whose velocity is faster than the other one is calculated is calculated for Hypothesis 2, expressed as $p 2$. Each observer's opinion on these two hypotheses can be reflected by $(p 1, p 2)$ which can be expressed by a point in a two-dimensional space. According to these points, the observers can be classified as different groups. In our study, the K-means clustering method was used. For better illustration, we define the term G-H1(Group of Hypothesis 1) to represent the observer group who voted more according to Hypothesis 1, which means relative disparity is predominant in determining visual discomfort. A similar definition is used for $\mathrm{G}-\mathrm{H} 2$. G-H12 is for the group who are equally sensitive to relative disparity and velocity, like the global subjective results. The clustering results are shown in Fig.6. The BT scores for all stimulus generated by each observer cluster are shown in Fig.7 and Fig.8, for experts and non-experts respectively.

It could be found that most of the observers agree with the global subjective experiment results. It's interesting to find that in G-H1 of experts, viewers perceived more visual discomfort with the increase of velocity when relative disparity was small. However, with the increase of the relative disparity, viewers felt more uncomfortable when velocity was slower. The experts who voted in this way gave the reasons that when the object's relative disparity was large but velocity was slow, it became difficult to fuse the foreground and the background at the same time, thus they would alternate the vergence between the two objects which made them more uncomfortable. But for fast velocity, they would not care about the other object as it appeared blurred due to its fast relative motion, which consequently reduced their visual discomfort. The G-H2 group in non-experts gave the opinion that the relative disparity as well as fast velocity were predominant factors in inducing visual discomfort.
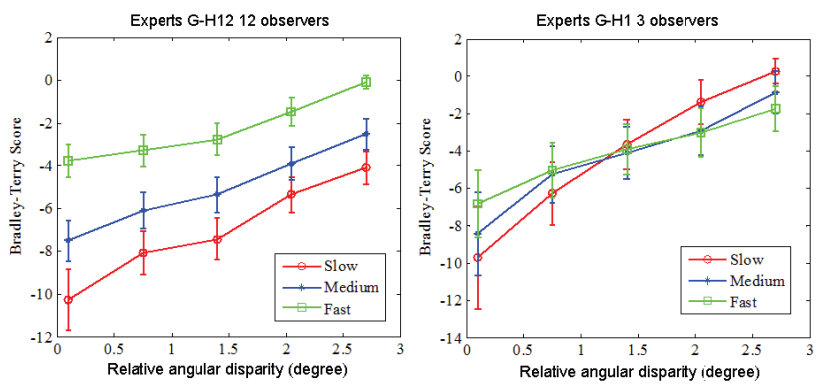

Fig. 7. The BT scores of visual discomfort for experts clusters. The left figure is for G-H12, and the right figure is for G-H1.

\section{CONCLUSIONS}

In this study, we investigated the effects of relative disparity and planar motion velocity on visual discomfort. The BT model was applied both on experts and non-experts subjective experiment data. The BT scores showed high agreement with our previous study. That is, the relative disparity between the foreground and background in the stimulus might be more significant in determining the visual discomfort than the binocular disparity of the foreground. Planar motion with faster velocity may result in more visual discomfort. We also evaluated the objective visual discomfort models which were developed in our previous work by the subjective data, the results showed that our models correlate quite well with the subjective perception.

As there were some observers who didn't agree with the global subjective experiment results, we classified these observers as different clusters according to which factor is predominant in determining their feeling of visual discomfort. The clustering results showed that most of the observers agreed with the global subjective experiment results. However, there were indeed some observers who considered either the relative disparity or velocity as the predominant factor in inducing visual discomfort while the other factor has small influence on their feelings.

The scope of the currently proposed model is limited to one foreground object with a circular motion on a structured background. In future work, some other factors which might also have influence in visual discomfort and a generalized model for predicting the visual discomfort induced by the natural scene stereoscopic images will be studied.

\section{ACKNOWLEDGEMENT}

The participants of the subjective experiment are gratefully acknowledged. This work has been partly conducted within the scope of the JEDI (Just Explore Dimension) ITEA2 project which 

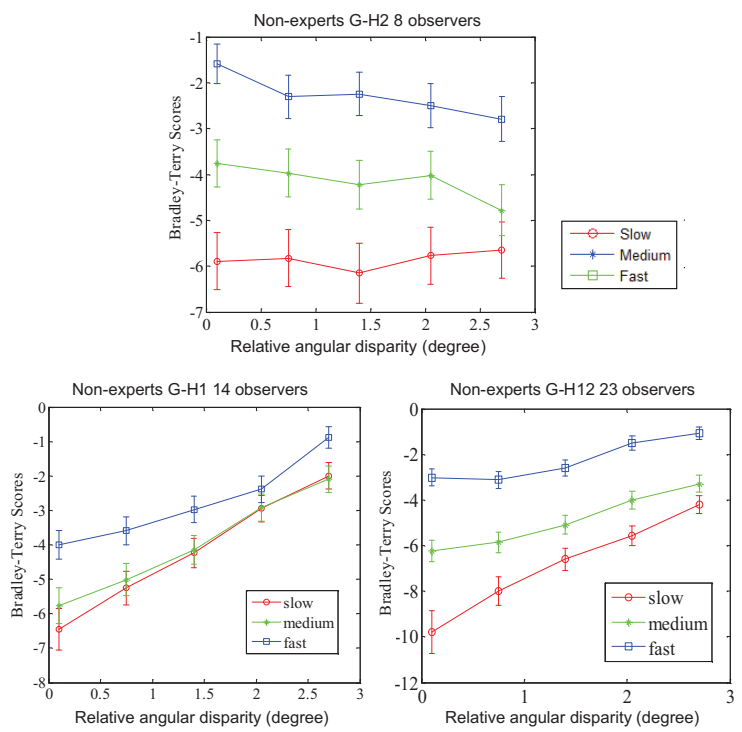

Fig. 8. The BT scores of visual discomfort for non-expert clusters. The figures at top, bottom-left and bottom-right represent G-H2,G-H1 and G-H12.

is supported by the French industry ministry through DGCIS and the PERSEE project which is financed by ANR (project reference: ANR-09-BLAN-0170).

\section{REFERENCES}

[1] D.M. Hoffman, A.R. Girshick, K. Akeley, and M.S Banks, "Vergence-accommodation conflicts hinder visual performance and cause visual fatigue," Journal of Vision, vol. 8, no. 3, pp. 1-30, 2008.

[2] F. L. Kooi and A. Toet, "Visual comfort of binocular and 3D displays," Displays, vol. 25, no. 2-3, pp. 99-108, Aug. 2004.

[3] S. Yano, S. Ide, T. Mitsuhashi, and H. Thwaites, "A study of visual fatigue and visual comfort for 3D HDTV/HDTV images," Displays, vol. 23, no. 4, pp. 191-201, Sept. 2002.

[4] S. Yano, M. Emoto, and T. Mitsuhashi, "Two factors in visual fatigue caused by stereoscopic HDTV images," Displays, pp. 141-150, Nov. 2004.

[5] F. Speranza, W. J. Tam, R. Renaud, and N. Hur, "Effect of disparity and motion on visual comfort of stereoscopic images," in Proc. of SPIE, 2006, vol. 6055, pp. 94-103.
[6] S. Lee, Y. J. Jung, H. Sohn, Y. M. Ro, and H. W. Park, "Visual discomfort induced by fast salient object motion in stereoscopic video," SPIE Stereoscopic Displays and Applications XXII, vol. 7863, 2011.

[7] J. Li, M. Barkowsky, J. Wang, and P. Le Callet, "Study on visual discomfort induced by stimulus movement at fixed depth on stereoscopic displays using shutter glasses," 17th International Conference on Digital Signal Processing, 2011.

[8] W. Chen, J. Fournier, M. Barkowsky, and P. Le Callet, "New requirements of subjective video quality assessment methodologies for 3DTV," Fifth International Workshop on Video Processing and Quality Metrics, 2010.

[9] N. Holliman, "3D display systems," in Handbook of optoelectronics, J. P. Dakin and R.G.W.Brown, Eds. Taylor \& Francis, London, 2006.

[10] D. A. Rosenbaum, Human Motor Control, Elsevier Inc., sec. edition, 2009.

[11] D. H. Brainard, "The psychophysics toolbox," Spatial Vision, vol. 10, pp. 443-446, 1997.

[12] ITU-R Recommendation BT.500, "Methodology for the subjective assessment of the quality of television pictures," 1974-2004.

[13] G. A. Gescheider, Psychophysics: The Fundamentals, chapter 9, Lawrence Erlbaum Associates, Mahwah, NJ., 3 edition, 1997.

[14] L. L. Thurstone, "Psychophysical analysis," American Journal of Psychology, vol. 38, pp. 368-389, 1927.

[15] L. L.Thurstone, "A law of comparative judgment," Psychological Review, vol. 101, no. 2, pp. 266-270, 1994.

[16] R. A. Bradley, "Paired comparisons: some basic procedures and examples," in Handbook of statistics, P. R. Krishnaiah and P. K. Sen, Eds., vol. 4, pp. 299-326. Elsevier science publishers, 1984.

[17] J. C. Handley, "Comparative analysis of Bradley-Terry and Thurstone-Mosteller paired comparison models for image quality assessment," in Proc. IS\&Ts Image Processing, Image Quality, Image Capture, Systems Conference, 2001, pp. 108-112.

[18] F. Wickelmaier and C. Schmid, "A Matlab function to estimate choice model parameters from paired-comparison data," Behavior Research Methods, Instruments, \& Computers, vol. 36, no. 1, pp. 29-40, Feb. 2004. 